\title{
Cambios en la estructura de la comunidad de anuros (Amphibia: Anura) en el Cerro Chompipe, Costa Rica
}

\author{
Juan G. Abarca Alvarado \\ Escuela de Ciencias Exactas y Naturales, Universidad Estatal a Distancia, San José. \\ Escuela de Ciencias Biológicas, Universidad Nacional, Heredia, Costa Rica; antinosedal@yahoo.es
}

Recibido 31-VIII-2011 Corregido 27-X-2011 Aceptado 3-XII-2011

\begin{abstract}
Changes in anuran (Amphibia: Anura) community composition in Cerro Chompipe, Costa Rica. Amphibian diversity in Costa Rica has declined over the past 40 years, mostly at higher elevations. Cerro Chompipe in the Central Volcanic Cordillera has suffered an extraordinary loss of these species. To measure the degree of contemporary impacts and their effects on anuran communities, I conducted diurnal and nocturnal amphibian surveys for one year at Cerro Chompipe and surrounding sites (from February 2010). Data were compared with species lists reported from the literature in the 1970s. In this survey I recorded only 7 of the 18 species reported originally for Cerro Chompipe, representing a 61\% loss of diversity. The most common species recorded were Craugastor podiciferus, Diasporus hylaeformis, Isthmohyla picadoi, I. pseudopuma, Incilius holdridgei and Lithobates taylori. In this survey, the presence of I. holdridgei, a critically endangered toad, was confirmed. Different species composition was observed at lower elevation sites then recorded previously, and none of the historic highland species were recorded at lower elevations. The tree frog, I. pseudopuma, occupied sites voided by species recorded previously, underscoring the observation that this species is an excellent colonizer because of their resistance and adaptation. In this paper I discuss how mass extinctions may alter and rearrange the trophic relationships in ecosystems.
\end{abstract}

\section{KEY WORDS}

Amphibians, diversity, extinction, recolonization, Cerro Chompipe, Costa Rica.

\section{RESUMEN}

La diversidad de anfibios ha disminuido en los últimos 40 años, principalmente en las zonas altas. El Cerro Chompipe, ubicado en la Cordillera Volcánica Central ha sufrido una pérdida considerable de especies. Para medir el grado de afectación de la comunidad se realizaron búsquedas directas durante un año, determinando la diversidad de anfibios diurnos y nocturnos. Los datos se compararon con la lista de especies reportadas en la literatura, desde 1970. Se contabilizaron solamente 7 especies, de las 18 reportadas para el Cerro Chompipe, esto representó una pérdida del $61 \%$ de la diversidad, las especies más comunes fueron Craugastor podiciferus, Diasporus hylaeformis, Isthmohyla picadoi, Isthmohyla pseudopuma, Incilius holdridgei y Lithobates taylori. Se constató la presencia del I. holdridgei, especie en peligro critico de extinción. En los sitios de menor altitud se observó una diferente composición de especies; por su parte la rana arbórea I. pseudopuma es una especie colonizadora que gracias a su resistencia ha venido a ocupar los sitios que dejaron las otras especies no encontradas. Se discute cómo las extinciones masivas pueden alterar y reorganizar las relaciones tróficas en los ecosistemas, al determinar los tamaños poblacionales se podrá entender mejor el proceso de intercambio y recolonización de especies.

\section{PALABRAS CLAVE}

Incilius holdridgei, Isthmohyla pseudopuma, extinción, recolonización, Cerro Chompipe, Costa Rica.
Costa Rica posee una enorme riqueza herpetológica, cerca de 433 especies de anfibios y reptiles (Bolaños et al. 2010a), siendo uno de los países mejor estudiados del mundo (Wake 2005). Como grupos indicadores los anfibios han sido utilizados en muchos campos para evaluar el grado de alteración de un bosque por las actividades humanas (Pineda et al. 2005), debido a sus especiales requerimientos ecológicos, íntimamente relacionados con la humedad y los ambientes acuáticos (Savage 2002, Young et al. 2004).

Globalmente se ha dado una disminución de anfibios en muchos lugares tanto alterados como prístinos (Pounds 2001), durante la década de los años 80 's y 90 's se reportaron extinciones masivas de especies en varios sitios, entre 
ellos El bosque Nuboso Monteverde y la Zona Protectora Las Tablas (Pounds 1997). Las extinciones locales han afectado a las especies de zonas altas y han tenido una menor repercusión en especies de zonas bajas (Pounds et al. 2006). Estas extinciones se han atribuido a la presencia de un hongo quitridio que afecta la epidermis de los anfibios (Lips et al. 2003), al cambio climático (Pounds 1997, 2001), efectos sinérgicos entre ambas (Pounds et al. 2006) o a factores desconocidos asociados con la salud de cada especie (G. Alvarado com. pers).

La comunidad científica preocupada por la pérdida de especies ha direccionado sus esfuerzos hacia la conservación de los anfibios, gracias a ello la Unión Internacional para la Conservación de la Naturaleza (UICN) realizó talleres de anfibios en el país en los años 2006, 2007 y 2010, en los cuales se actualizaron las listas rojas y se establecieron nuevas estrategias de conservación (Bolaños et al. 2010b).

Las especies costarricenses dentro de las categorías más amenazadas según la UICN 2011 son Extintas: Incilius holdridgei, I. periglenes, Craugastor escoces, En Peligro crítico: Atelopus varius, A. chiriquiensis, A. senex, I. fastidiosus, Duellmanohyla uranochroa, Agalychnis lémur, Hyloscirtus colymba, Isthmohyla angustilineata, I. calypsa, I, debilis, I. graceae. I. rivularis, I. tica, Craugastor andi, C. angelicus, C. catalinae, C. emcelae, C. fleischmanni, C. ranoides, C. taurus, Lithobates vibicarius, Nototriton major, Oedipina altura, $O$. paucidentata (UICN 2011).

En los talleres mencionados se sugirió la necesidad de iniciar programas de reproducción y seguimiento cuando se definieran poblaciones conocidas de alguna de las especies en los sitios donde se les observaba. Uno de esos sitios es el Cerro Chompipe, en la montañas de Heredia, allí no se ha revisado el cambio en la comunidad de anfibios y los reportes de desapariciones aunque claros, son anecdóticos; ya que este era uno de los sitios más cercanos donde se observaban muchísimas ranas y poco a poco se dejaron de observar (G. Chávez com. pers.).

La presente investigación es parte de un esfuerzo nacional de conservación de anfibios en peligro de extinción, el objetivo principal fue determinar la diversidad actual de anfibios en el Cerro Chompipe, y otras partes cercanas de la Cordillera Volcánica Central, para medir el aparente descenso en la composición de la comunidad de anfibios a lo largo de las últimas 4 décadas.

\section{METODOLOGÍA}

El sitio principal de estudio se encuentra ubicado en el Noroeste de la provincia de Heredia, en el cantón de San Rafael y Vara Blanca, al Este de las faldas del Volcán Barva, $\left(10.09^{\circ} \mathrm{N}, 84.06^{\circ} \mathrm{W}\right)$ en la base del Cerro Chompipe, en este sector se encuentran varias areas protegidas como lo son los Refugios de Vida Silvestre Cerro Dantas y Jaguarundi, los cuales se encuentran dentro de la Reserva Forestal Cordillera Volcánica Central y cerca del Parque Nacional Braulio Carrillo (Fig. 1). La región pertenece a la zona de vida del Bosque Tropical Pluvial Montano Bajo (Tosi 1969), sin estación seca definida (Herrera 1985), la precipitación es de $3000 \mathrm{~mm}$ anuales, la temperatura ambiental oscila entre $15^{\circ}$ y $18^{\circ} \mathrm{C}$ (Segura 1990).

La principal actividad económica local es la ganadería y el turismo, además existen muchas fincas que están sometidas a pagos de servicios ambientales y en sus faldas nacen varios ríos importantes como el Río Segundo y el Río Patria. Para complementar la investigación se realizaron dos visitas a otros sitios montañosos cercanos dentro del Parque Nacional Braulio Carrillo: Puesto Zurquí, Bajo de la Hondura y Quebrada González.

Los muestreos se realizaron durante dos días cada mes entre febrero del 2010 y febrero del 2011, para inventariar las especies actuales de ranas en la zona de estudio se utilizaron transectos con encuentros visuales (Crump \& Scott 2001), búsqueda auditiva nocturna y diurna (Zimmerman 2001). Para georeferenciar los sitios se utilizó un GPS Garmin72. Para determinar las especies que se encontraban en el pasado y el cambio en la composición en estos sitios se obtuvieron dos fuentes de referencia a Savage (2002) y a Novak \& Robinson (1975), además se consultaron las listas de colecta del Museo de Zoología de la Universidad de Costa Rica.

Los lugares principales del Cerro Chompipe donde se encontraron ranas de forma abundante fueron: partiendo del Refugio de Vida Silvestre Cerro Dantas (Punto A: 10 $05^{\prime} 40^{\prime \prime} \mathrm{N} ; 8^{\circ} 03^{\prime} 30^{\prime \prime} \mathrm{O}$ ), hacia el oeste el camino que comunica el Río las Vueltas (Punto B: $10^{\circ} 05^{\prime} 30^{\prime \prime} \mathrm{N} ; 8^{\circ} 03^{\prime} 57^{\prime \prime} \mathrm{O}$ ) con Alto del Roble (Punto C: $10^{\circ} 05^{\prime} 13^{\prime \prime} \mathrm{N}$; 84 $4^{\circ} 04^{\prime} 19^{\prime \prime} \mathrm{O}$ ) y zonas aledañas. Entre el camino que comunica el Alto del Roble con la escuela las Nubes (Punto D: $10^{\circ} 05^{\prime} 11^{\prime \prime} \mathrm{N}$, $\left.84^{\circ} 04^{\prime} 45^{\prime \prime} \mathrm{O}\right)$ y el camino hacia el norte después de la escuela las Nubes, $2 \mathrm{~km}$ al norte del punto C, cerca de los límites del refugio Jaguarondi (Punto E: $10^{\circ} 05^{\prime} 46^{\prime \prime} \mathrm{N}$; 840.'30"O) (Fig. 2.)

Los otros sectores que se visitaron en las zonas aledañas del Parque, fueron el Puesto Zurquí, Bajo de la Hondura y Quebrada González en la Ruta 32 (Fig.1) estos se visitaron para complementar la composición de especies para el Parque Braulio Carrillo, sin embargo, para la comparación histórica se tomaron únicamente las localidades de Alto del Roble y Cerro Chompipe mencionados por Novak \& Robinson (1975). 


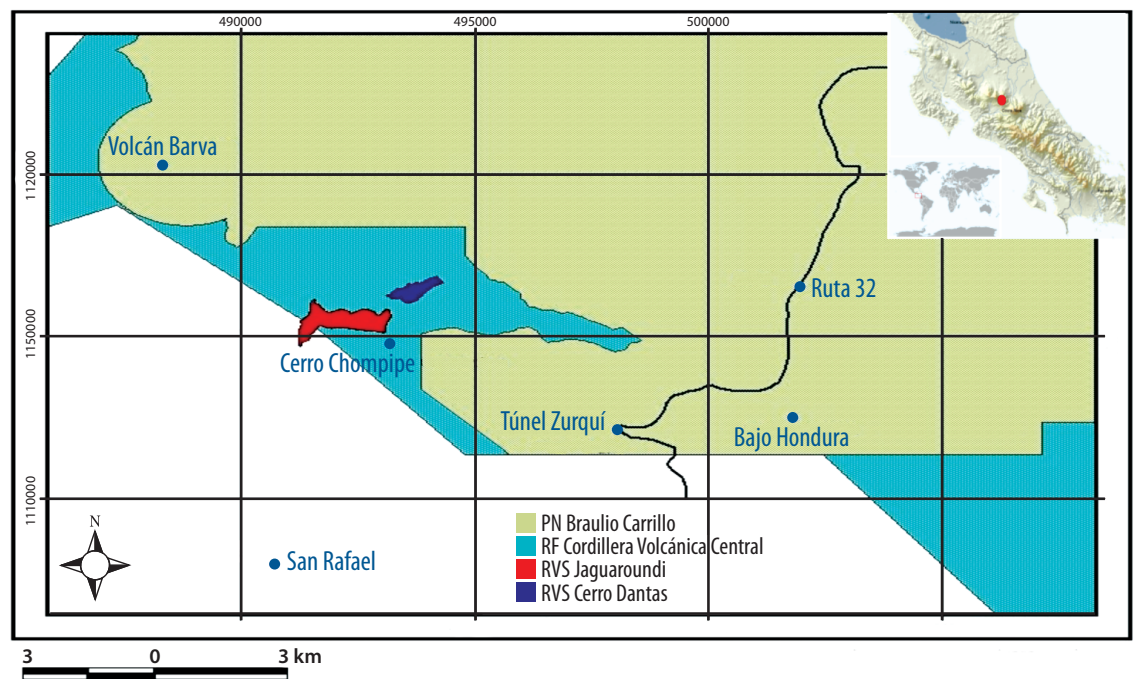

FIG. 1. Áreas Protegidas de la Cordillera Volcánica Central, mostrando la ubicación del Cerro Chompipe y localidades aledañas.

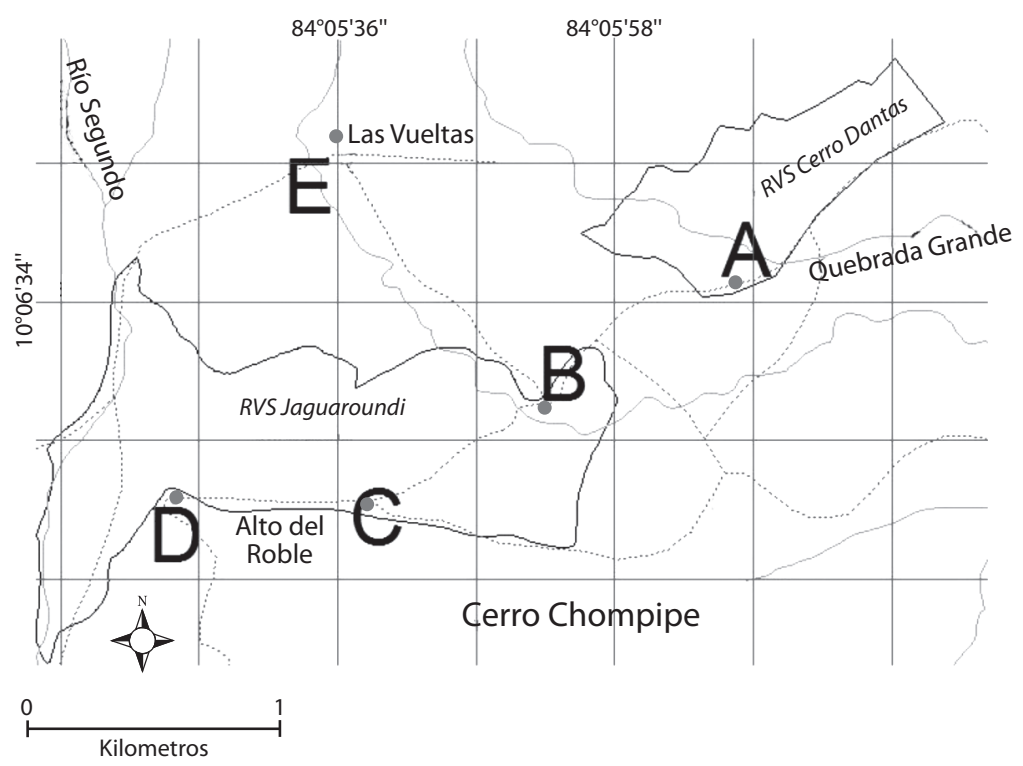

FIG. 2. Puntos de muestreo principales en la base del Cerro Chompipe donde se ubicaron las especies. 


\section{RESULTADOS}

El cuadro 1 muestra 18 especies de ranas que se podían encontrar usualmente en el Cerro Chompipe, o que se han reportado que se distribuyen a esa altitud, según su hábitat preferencial la mayoría eran especies arbóreas asociadas a ríos o terrestres asociadas a ríos, de ellas solamente 6 se encuentran en preocupación menor, el resto están incluidas en categorías de alto riesgo de extinción.
Durante el periodo de estudio (Febrero 2010- febrero 2011) en el Cerro Chompipe se encontraron únicamente la siguientes especies: C. podiciferus, C. melanostictus, Diasporus hylaeformis, I. picadoi, I. pseudopuma, L. taylori, I. holdridgei. Otros datos recientes de observación mencionan para esta zona a Crepidophryne chompipe (Vaughan \& Mendelson 2007) y para la ladera Este del Volcán Barva a I. angustilineata (Nishida 2006), los datos de I. rivularis y L. vibicarius están aún por confirmarse.

CUADRO 1

Comparación de datos históricos de presencia de las especies de anfibios según los autores, Novak \& Robinson 1975 (A) y Savage 2002 (B) con las especies encontradas en este estudio.

\begin{tabular}{|c|c|c|c|c|}
\hline Familia / Especie & Autor & Habito & Categoría actual UICN & $\begin{array}{c}\text { Puntos de } \\
\text { observación } 2010\end{array}$ \\
\hline \multicolumn{5}{|l|}{ Hylidae } \\
\hline Isthmohyla angustilineata & $A-B$ & Arbórea, zona abierta. & En peligro crítico & - \\
\hline Isthmohyla pictipes & B & Arbórea, ripario & En peligro & - \\
\hline Isthmohyla rivularis & $\mathrm{B}$ & Arbórea, ripario & En peligro crítico & $B^{*}$ \\
\hline Isthmohyla tica & B & Arbórea, ripario & En peligro crítico & - \\
\hline Isthmohyla xanthosticta & $\mathrm{B}$ & Arbórea, ripario & En peligro crítico & - \\
\hline Isthmohyla pseudopuma & $\mathrm{B}$ & Arbórea, terrestre, zona abiertas & Preocupación menor & $A, B, C, D, E$ \\
\hline Isthmohyla picadoi & B & Arbórea, bromelias & Preocupación menor & $A, B, C, D$ \\
\hline Ecnomiohyla fimbrimembra & B & Arbórea, bosque & En peligro & - \\
\hline \multicolumn{5}{|l|}{ Bufonidae } \\
\hline Atelopus senex & $A-B$ & Terrestre, ripario & En peligro crítico & - \\
\hline Atelopus varius & $A-B$ & Terrestre, ripario & En peligro crítico & - \\
\hline Incilius holdridgei & $A-B$ & Terrestre, zonas abiertas & En peligro crítico & $C, E$ \\
\hline \multicolumn{5}{|l|}{ Craugastoridae } \\
\hline Craugastor escoses & $A-B$ & Terrestre, ripario & Extinto & - \\
\hline Craugastor podiciferus & $A-B$ & Terrestre, hojarasca & Preocupación menor & $A, B, C, E$ \\
\hline Craugastor melanostictus & B & Terrestre, ripario & Preocupación menor & $\mathrm{B}, \mathrm{E}$ \\
\hline Craugastor fleischmanni & B & Terrestre, ripario & En peligro crítico & - \\
\hline \multicolumn{5}{|l|}{ Eleutherodactylidae } \\
\hline Diasporus hylaeformis & B & Arbórea, áreas abiertas & Preocupación menor & $A, B, C, D$ \\
\hline \multicolumn{5}{|l|}{ Ranidae } \\
\hline Lithobates taylori & $A-B$ & Terrestre, zonas abiertas & Preocupación menor & $\mathrm{C}$ \\
\hline Lithobates vibicarius & $A-B$ & Terrestre, zonas abiertas & En peligro crítico & $A^{*}$ \\
\hline
\end{tabular}


Con respecto a las otras localidades (Fig. 3) en el sitio de Quebrada González, dos visitas en julio y septiembre, permitieron constatar la presencia de 8 especies: $C$. fitzingeri, L. warszewitschii, I. melanochlorus, Rhaebo haematiticus, I. pseudopuma, D. diastema, C. bransfordii, Smilisca phaeota. Estas especies son comunes y todas están en la categoría de preocupación menor, de la lista roja de la UICN (2011). Para el sector Zurquí solamente se registró la presencia de C. underwoodi y en Bajo de la Hondura, se encontró C. fitzingeri, I. pseudopuma, Duellmanohyla rufioculis y L. taylori.

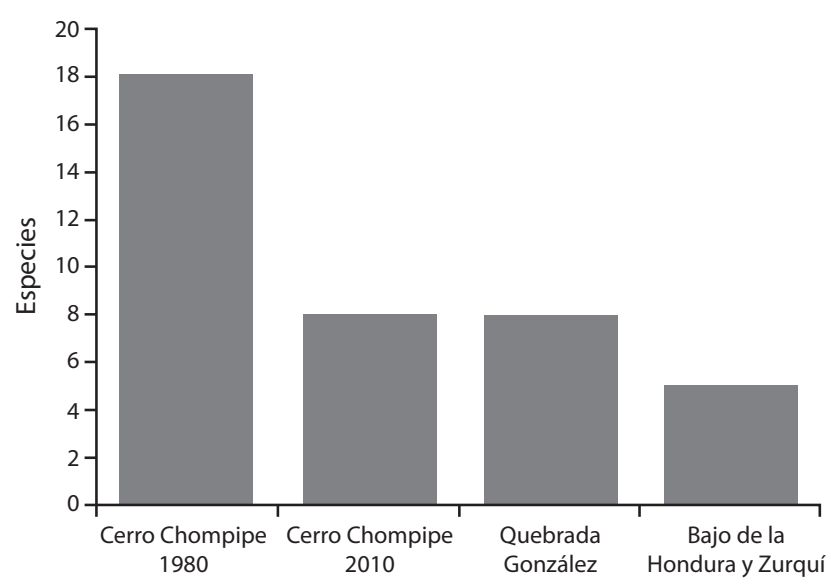

FIG. 3. Número de especies de anuros en Cerro Chompipe y zonas aledañas.
Para todos los sitios en conjunto se obtuvieron un total de 18 especies, en comparación con las 95 reportadas para todo el Parque Braulio Carrillo (Tenorio 2007), esto significa un $18.9 \%$ de la diversidad del Parque, sin embargo, estos datos corresponden a todo el parque que incluye el sector de La Finca la Selva, la cual se encuentra en una zona de menor altitud. Aunque los sitios de menor altitud tuvieron un esfuerzo de muestreo menor, se observaron especies comunes que no están en una categoría de riesgo de la IUCN (IUCN 2011), pero casi ninguna de ellas es parte de la comunidad de anfibios de Cerro Chompipe, solamente I. pseudopuma y L. taylori. El número de especies por sector muestra como se dio una reducción drástica en el Cerro Chompipe reflejando que en el pasado era un sitio altamente diverso.

Durante el 2008 se encontraron varias especies no identificadas, el año pasado se confirmó la presencia de la especie Incilius holdridgei (Abarca et al. 2010). Durante el presente muestreo esta especie se encontró en el Alto del Roble, en el camino de ingreso y en los punto $\mathrm{C}$ y $\mathrm{E}$ (Fig. 4). Esta representa la primera especie importante para la conservación y aplicación de un programa de reproducción en cautiverio, el siguiente paso para su conservación sería determinar un estimado de la población y los puntos importantes que representen sitios de puestas y reproducción.

Además de esta especie, otras que se consideraban con poblaciones reducidas se han reportado cerca de este lugar, como I. angustilineta que fue encontrada $9 \mathrm{~km}$ noroeste de este sitio, se menciona también que algunos estudiantes observaron C. cf. fleischmanni (Zumbado et al. 2011) en el sector de Sacramento y otra fue encontrada cerca del Parque Nacional Volcán Poas. Otra especie significativa es $C$. melanostictus ya que presenta una ecología

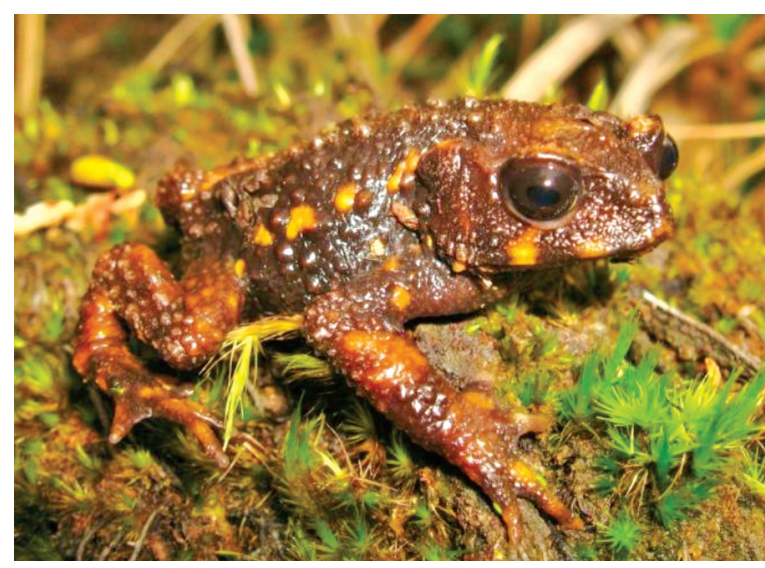

FIG. 4. Incilius holdridgei. 
asociada a los ríos similar a las especies del grupo C. rugulosus, incluso se observaron cerca de riachuelos donde los Atelopus, ahora desaparecidos, eran muy abundantes.

Las especies restantes encontradas en los otros sitios son comunes y se encuentran en preocupación menor en la lista de la UICN (2011). Un caso especial es el de I. pseudopuma (Fig. 5) que no era observada con frecuencia antes de los años ochenta. Novak y Robinson (1975) no las incluyen en sus listados y en el Museo de Zoología de la UCR no se tienen colectas de la especie en este sitio sino a partir de los años 90 después de que muchas otras especies se dejaron de observar. Hoy en día esta especie parece ser sumamente común, esto porque fue encontrada en todos los sitios de muestreo tanto en Cerro Chompipe como en las otras zonas más bajas.

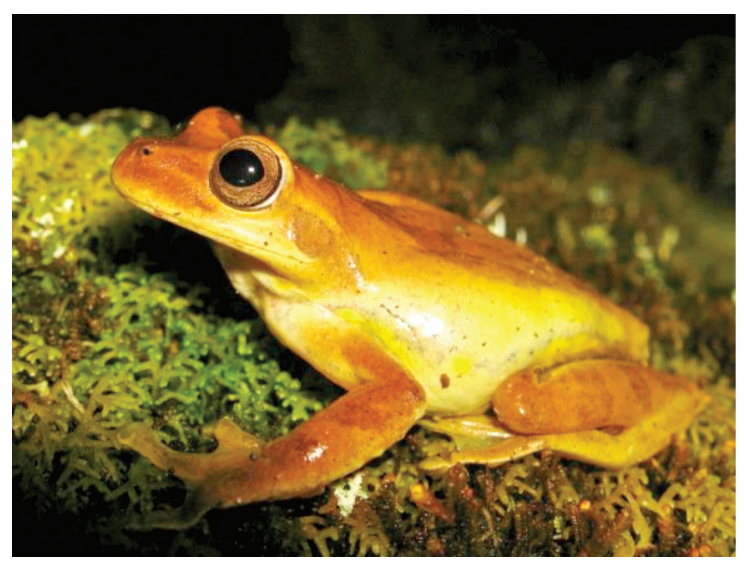

FIG. 5. Isthmohyla pseudopuma.

Si analizamos los sitios de puesta de I. holdridgei mostrados por Novak \& Robinson (1975), estos abarcaban todas las fuentes de agua que se encontraban desde el punto $A$ hasta el $D$ (Fig. 2) pero actualmente esa especie está reducida a dos localidades marcadas con los puntos $\mathrm{C}$ y E. Por el contrario I. pseudopuma, que estaba totalmente ausente, hoy día utiliza esos mismos sitios reproductivos. I. pseudopuma ha llegado a ocupar los sitios que dejaron las ranas arbóreas (Hylidae) y hojarasqueras (Craugastoridae), como lo son las posas temporales, los árboles y la hojarasca. Aunque aún no ha podido colonizar las áreas de ríos, este evento muestra como en la actual extinción está ocurriendo lo que se había previsto: que algunas pocas especies comunes y resistentes vendrían a reemplazar y a tomar el puesto de aquellas que están desapareciendo (McKinney \& Lockwood 1999).

La pérdida considerable de especies puede provocar alteraciones en el ecosistema, por ejemplo en el caso de los anfibios, debido a que son tanto depredadores como presas, su disminución puede provocar una inestabilidad en sectores de la red trófica, con consecuencias desconocidas en el comportamiento, alimentación o estructura poblacional de otras especies (Solé et al. 2002). Por ejemplo, en el caso de las serpientes con una dieta especializada en ranas, una disminución notable de presas puede afectar indirectamente las poblaciones y el estado de salud de las mismas; las consecuencias indirectas que el fenómeno provoca hacia otros grupos aún no han sido consideradas en la investigación de desapariciones de anfibios.

Este escenario de múltiples extinciones locales de ranas, nos pone frente a un proceso de extinción masiva, cuando esto ocurre los ecosistemas se reorganizan por entero (Bowring et al. 1999). Este fenómeno, que solo se conocía al estudiar los fósiles, tiene una fuerza comparada con la selección natural ya que provoca una radiación masiva de nuevas especies que ocupan los nichos que han quedado disponibles (Solé et al. 2002). La desaparición de especies abre la posibilidad de que una especie altamente resistente llegue a colonizar nuevos sitios, según Scott (1976) la especie más común es la que se ha especializado en obtener el recurso más abundante y en la mayoría de los casos la especie más abundante es también la más resistente.

El Cerro Chompipe en el pasado era un sitio de gran abundancia y diversidad de anfibios, igual o mayor que la que existe hoy en las zonas bajas, por tanto presenta una notable desaparición de especies. Asimismo, si incluimos a las encontradas en el 2011, de las sobrevivientes 3 están en Peligro Crítico, 3 en Peligro y 7 Preocupación menor (IUCN 2011). El siguiente paso en esta investigación es estimar el tamaño poblacional de estas especies, caracterizar el hábitat de cada una, la frecuencia de observación y buscar sitios de reproducción. Se recomienda utilizar esas especies en peligro para programas de monitoreo y aumentar los esfuerzos de conservación en las áreas protegidas y otros puntos geográficos dentro del Parque, esto permitirá conocer mejor el panorama actual de las poblaciones de anfibios en la Cordillera Volcánica Central.

\section{AGRADECIMIENTOS}

Esta investigación se realizó gracias al apoyo del Sistema Nacional de Áreas de Conservación del Ministerio de Ambiente, Energía y Telecomunicaciones (SINAC-MINAET), resolución No: PI-ACCVC 012-2010. Agradezco al director Rolando Vargas González y al coordinador técnico Rodolfo Tenorio del Parque Nacional Braulio CarrilloACCVC. Agradezco también a Warren Calvo del Refugio de Vida Silvestre Cerro Dantas, a Johnny Villareal Orias de la UNED, a Heinz Hoffmann, Víctor Acosta y Michael Méndez 
por el apoyo en el monitoreo de campo; a Gerardo Chávez y Adrián García del Museo de Zoología de la Universidad de Costa Rica. Gilbert Alvarado, Oscar Ramírez Allan y a dos revisores anónimos por los comentarios al manuscrito.

\section{REFERENCIAS}

Abarca, J., G. Chaves, A. García \& R. Vargas. 2010. Reconsidering Extinction: Rediscovery of Incilius holdridgei (Anura: Bufonidae) in Costa Rica After 25 Years. Herpetologial Review 41: 150-152.

Bolaños, F., J. M. Savage \& G. Chaves. 2010a. Anfibios y Reptiles de Costa Rica. Listas Zoológicas Actualizadas Museo de Zoología UCR. UCR: (http://museo.biologia.ucr. ac.cr/Listas/LZAPublicaciones.htm; consultado el 18 de octubre, 2011).

Bolaños, F., G. Chaves, J. E. Rodríguez, B. Young \& Y. Matamoros (Eds.) 2010b. Taller para revisar la lista roja de anfibios de Costa Rica de la UICN y evaluación del cumplimiento de las acciones de la estrategia de conservación de los anfibios de Costa Rica. 3-4 de agosto, 2010. Escuela de Biología de la Universidad de Costa Rica, San Pedro, San José, Costa Rica. Conservation Breeding Specialist Group (SSC/IUCN)/CBSG Mesoamerica. (http://www.cbsg.org/ cbsg/workshopreports/26/anfibios.pdf; consultado 18 octubre, 2011).

Bowring, S. A., D. H. Erwin \& Y. Isozaki. 1999. The tempo of mass extinction and recovery: The end-Permian example. Proceedings of the National Academy of Sciences 96: 8827-8828.

Crump, M. L. \& N. J. Scott. 2001. Relevamientos por encuentros visuales. In W. R Heyer, M.A. Donnelly, R. W. McDiarmid, L.A. Hayek \& M. S. Foster (eds.). Medición y monitoreo de la diversidad biológica: métodos estándarizados para anfibios. Smithsonian Institution Press-Editorial Universitaria de la Patagonia, Washington y Londres.

Herrera, W. 1985. Clima de Costa Rica. EUNED, San José, Costa Rica.

Lips, K. R., J. D. Reeve \& L. R. Witters. 2003. Ecological traits predicting amphibian population declines in Central America. Conservation Biology 17: 1078-1088.

McKinney M. L. \& J. L. Lockwood. 1999. Biotic homogenization: a few winners replacing many losers in the next mass extinction. TREE 14: 450-453.

Nishida, K. 2006. Encounter with Hyla angustilineata Taylor 1952 (Anura,Hylidae) in a cloud forest of Costa Rica. Brenesia 66: 79-81.

Novak, R. \& D. C. Robinson. 1975. Observations on the reproduction and ecology of the tropical montane toad, Bufo holdridgei Taylor in Costa Rica. Revista de Biología Tropical 23: 213-237.

Pineda, E., C. Moreno, F. Escobar \& G. Halffter. 2005. Frog, bat, and Dung Beetle diversity in the cloud forest and coffee agroecosystems of Veracruz, Mexico. Conservation Biology 19: 400-410.

Pounds, J. A. 1997. Golden toads, null models, and climate change. Froglog 1: 2.

Pounds, J. A. 2001. Climate and amphibian declines. Nature 410: 639-640.

Pounds, J. A., M. R. Bustamante, L. A. Coloma, J. A. Consuegra, M. P. Fogden, P. N. Foster, E. La Marca, K. L. Masters, A. M. Vitieri, R. Puschendorf, S. R. Ron, G. A. Sánchez, C. J. Still \& B. Young. 2006. Widespread amphibian extinctions from epidemic disease driven by global warming. Nature 439: 161-167.

Savage, J. M. 2002. The amphibians and reptiles of Costa Rica. A herpetofauna between two continents, and two seas. The University of Chicago, Chicago, USA.

Scott, N. J. 1976. The abundance and diversity of the herpetofaunas of tropical forest litter. Biotropica 8: 41-58.

Segura, P. 1990. Historia San Rafael de Heredia. Consejo Municipal, San Rafael. Mimeografiado.

Solé, R. V., J. M. Montoya \& D.H. Erwin. 2002. Recovery After Mass Extinction: Evolutionary assembly in large scale biosphere dynamics. Philosophical Transactions of the Royal Society 357: 697-707.

Tenorio, R. 2007. Parque Nacional Braulio Carrillo un viaje a la biodiversidad. SINAC-MINAET. (http://www.cientec.or.cr/ exploraciones/ponencias2007/RodolfoTenorio-BraulioCarrillo.pdf; consultado 28 agosto, 2011).

Tosi, J.A. 1969. Mapa ecológico, República de Costa Rica: según la clasificación de las zonas de vida del mundo de L. R. Holdrigde. Centro Científico Tropical, San José, Costa Rica.

UICN. 2011. IUCN red list of threatened species. UICN. (www.iucnredlist.org; consultado el 20 de octubre, 2011).

Vaughan, A. \& J. R. Mendelson. 2007. Taxonomy and ecology of the central american toads of the genus Crepidophryne (Anura: Bufonidae). Copeia 2007: 304-314.

Wake, D.B. 2005. Diversity of Costa Rican salamanders, p. 65-80, In M.A. Donnelly, B.I. Crother, C. Guyer, H.W. Marvalee \& M.E. White (eds.). Evolution and ecology in the tropics. The University of Chicago, Chicago, USA.

Young, B. E., S. N. Stuart, J. S. Chanson, N. A. Cox, \& T. M. Boucher. 2004. Disappearing Jewels: The status of NewWorld amphibians. NatureServe, Arlington, Virginia, USA.

Zimmerman, B. L. 2001. Transectos de bandas auditivas, p. 87-93, In W. R. Heyer, M.A. Donnelly, R. W. McDairmid, L. A. Hayek \& M. S. Foster (eds.). Medición y monitoreo de la diversidad Biológica: Métodos estandarizados para anfibios. Editorial Universitaria de la Patagonia, Chubut, República de Argentina.

Zumbado, H., A. García, G. Chavez \& G. Alvarado. 2011. Searching for lost frogs of the Craugastor rugulosus group: understanding their dissapearence and assessing their current population status. Froglog 96: 28. 